\title{
Optimization of Short-term Modes of Hydrothermal Power System
}

\author{
Tulkin Gayibov ${ }^{*}$, Bekzod Pulatov ${ }^{2}$ \\ ${ }^{1,2}$ Tashkent State Technical University named after Islam Karimov, Uzbekistan
}

\begin{abstract}
Optimal planning of short-term modes of power systems is a complex nonlinear programming problem with many simple, functional and integral constraints in the form of equalities and inequalities. Especially, the presence of integral constraints causes significant difficulties in solving of such problem. Since, under such constraints, the modes of power system in separate time intervals of the considered planning period become dependent on the values of the parameters in other intervals. Accordingly, it becomes impossible to obtain the optimal mode plan as the results of separate optimization for individual time intervals of the period under consideration. And the simultaneous solution of the problem for all time intervals of the planning period in the conditions of large power systems is associated with additional difficulties in ensuring the reliability of convergence of the iterative computational process. In this regard, the issues of improving the methods and algorithms for optimization of short-term modes of power systems containing thermal and large hydroelectric power plants with reservoirs, in which water consumption is regulated in the short-term planning period, remains as an important task.

In this paper, we propose the effective algorithm for solving the problem under consideration, which makes it possible to quickly and reliably determine the optimal operating modes of the power system for the planned period. The results of research of effectiveness of this algorithm are presented on the example of optimal planning of daily mode of the power system, which contains two thermal and three hydraulic power plants..
\end{abstract}

\section{Introduction}

Considering hydroelectric power plants (HPPs) in the tasks of optimization the modes of power system, we mean the presence of a regulated reservoir with it, which allows it to work with a variable mode of power output in the context of the panned period. Hydroelectric power plants operating on the domestic flow of rivers, the capacities of which are unambiguously determined by the current inflows of water, are usually replaced by negative loads and do not participate in optimization. In the presence of large hydroelectric power plants with reservoirs and, accordingly, with given water flow rates, the modes of power systems for the time interval of the planning period become dependent and the procedure for optimization of their daily mode becomes more complicated.

Hydroelectric power plants play a large role in the operation of modern EPS: by removing the peak part of their loads, they allow reducing the installed capacity of TPPs. In addition, the presence of HPPs in the power system is essential for maintaining the balance of power in them in emergency modes, since HPPs are more mobile than thermal power plants (TPPs).
Usually, for hydroelectric power plants participating in the optimal planning of short-term modes of power systems, a limitation on water consumption associated with the need to maintain the required water level in reservoirs for irrigation or other purposes is established, Thus, the problem of optimal planning of short-term mode of power system containing HPPs with reservoirs is to determine such load schedules for all TPPs and HPPs, which ensure the minimum total fuel costs in TPPs, required water levels in HPP reservoirs, minimal impact on the environment and all other established operating and technological restrictions [1-4]. Accordingly, the problem under consideration is mathematically a complex nonlinear programming problem. It is characterized by the presence, in the general case, of many simple, functional and integral constraints [5-7]. Integral constraints exclude the use of short-term planning methods based on individual solutions to optimization problems for individual time intervals of the period under consideration. Since, due to integral limitations, the power system modes in certain time intervals become dependent on the parameters of other intervals. On the other hand, the simultaneous solution of such problem for all time intervals of the planning period is associated with special difficulties in ensuring the reliable convergence of iterative

\footnotetext{
* Corresponding author: tulgayibov@gmail.com
} 
calculation process to the solution with sufficient accuracy.

Many works of authors from all over the world are devoted to the issue of optimal planning of short-term modes of hydrothermal power system, who have made a significant contribution to the development of the theory and methods for solving the problem under consideration. These include a number of algorithms that implement various optimization methods such as Lagrange multipliers [8,9], gradient search [10, 11, $16]$, evolutionary programming $[12,13]$, genetic $[14$, 15], etc. etc. At the same time, the algorithms for solving the problem under consideration cannot be considered perfect. For example, the algorithms mentioned above based on the methods of Lagrange multipliers and gradient search are characterized by slow, and sometimes not reliable, convergence of the iterative calculation process. Evolutionary and genetic algorithms also suffer from poorer search performance. Thus, the issues of improving the methods and algorithms for optimization of short-term modes of power systems with large hydroelectric power plants participating in the optimization and regulation of reservoir modes during the planned periods remains an urgent task.

In this paper, an effective algorithm for solving the described problem, which is distinguished by a sufficiently fast and reliable convergence of iterative calculation process, is presented.

\section{The method and algorithm of optimization}

The problem of optimization of short-term, for example daily, mode of an energy system containing $n$ TPPs and $m$ HPPs with water flow control during the planning period, in the general case, can be represented as follows:

minimize the function of total fuel costs in TPPs per day

$$
B=\sum_{i=1}^{n} \sum_{t=1}^{24} B_{i t}\left(P_{i t}\right)
$$

subject to constraints:

by balance of active power in power system -

$$
\sum_{i=1}^{n} P_{i t}+\sum_{j=1}^{m} P_{j t}=P_{L t,} \quad t=1,2, \ldots 24 ;
$$

by available capacity of power plants -

$$
\begin{aligned}
& P_{i t}^{\min } \leq P_{i t} \leq P_{i t}^{\max }, \quad i=1,2, \ldots n, \quad t=1,2, \ldots 24 ; \\
& P_{j t}^{\min } \leq P_{j t} \leq P_{j t}^{\max }, \quad j=1,2, \ldots, m, \quad t=1,2, \ldots 24 ;
\end{aligned}
$$

on flows of active power in controlled power lines -

$$
P_{l t}^{\text {min }} \leq P_{l t} \leq P_{l t}^{\max }, \quad l=1,2, \ldots, L_{p}, \quad t=1,2, \ldots 24 ;
$$

by water consumption in HPPs per day

$$
\Delta Q_{j}=\sum_{t=1}^{24} Q_{j t}\left(H_{j t}, P_{j t}\right)-Q_{j}=0, \quad j=1,2, \ldots m .
$$

In (1) - (6) $B$ is total fuel costs in $n$ TPPs per day; $P_{i t}, P_{j t}$ are active loads of $i$ th TPP and $j_{\text {th }}$ HPP at the $t$ th hour of the day; $P_{L \mathrm{t}}$ is total load of power system consumers at the $t$ th hour of the day; $P_{i t}^{\min }, P_{j t}^{\min }, P_{i t}^{\max }, P_{j t}^{\max }$ are the minimum and maximum possible loads of the $i$ th TPP and the $j$ th HPP at the $\mathrm{t}$-th hour of the day (taking into account the optimal composition of operating units in power plants); $\mathrm{P}_{\mathrm{lt}}$ is the flow of active power into the 1th controlled power transmission line (PTL) at the t-th hour of the day; $P_{t t}^{\min }, P_{l t}^{\max }$ are the minimum and maximum possible flows of active power in the $l$ th controlled transmission line at the $t$ th hour of the day; $\mathrm{Lp}$ is the number of transmission lines in which the power flows are controlled; $H_{j t}, Q_{j t}$ are head and water flow rate in the $j$ th hydroelectric power station at the $t$ th hour of the day; $\mathrm{Q}_{\mathrm{j} 3}$ is a given volume of water in the $j$ th hydroelectric power plant, which should be optimally consumed during the day.

In the proposed algorithm, the solution to problem (1) (6) is reduced to minimizing the Lagrange function

$$
L=\sum_{i=1}^{n} B_{i}\left(P_{i}\right)+\sum_{j=1}^{m} \lambda_{j} \Delta Q_{j}
$$

subject to constraints (2) - (5). The indefinite factor $\lambda_{j}$ in (7) is physically the costly equivalent of the water consumption in the $j$ th hydroelectric power plant and is numerically equal to the amount of saved fuel costs at the thermal power plant with an increase in the daily water consumption in the $j$ th hydroelectric power plant by $1 \mathrm{~m}^{3}$. It remains constant during the regulation cycle [1].

At the known values of the factors $\lambda_{j}$, the solution of the problem is carried out as an optimization of mode of power system containing only the calculated TPPs, since multiplying the energy characteristics of each HPP by the corresponding factors $\lambda_{j}$ it is reduced, in the calculated sense, into the category of TPPs. In this case, the problem of optimal coverage of the daily load schedule of power system is reduced to sequential optimal distribution of power system load, for each interval of regulation cycle, between the calculated power plants, taking into account constraints (3) - (5). In this case, the objective function is represented in the form (7).

Thus, in the setting adopted here, the main problem is related to the determination of the values of the Lagrange multipliers $\lambda_{j}$. The presence of kinks and vertical-horizontal sections in the $\Delta \mathrm{Q}_{\mathrm{j}}\left(\lambda_{\mathrm{j}}\right)$ dependences complicates the use of methods using derivatives $\frac{\partial Q}{\partial \lambda}$ for this purpose.

The described algorithm for optimal planning of shortterm mode of power system provides for the determination of values of these factors $\lambda_{j}$ by $a$ combined algorithm based on the use of chord methods and simple selection in the process of iterative calculation. Below we describe the essence of the algorithm.

The choice of the initial values of the factors $\lambda_{j}{ }^{(0)}(j=$ $1,2, \ldots, \mathrm{m})$ is carried out by the method of average values as in [1]. Refinement of values of factors after the first iteration $\lambda_{j}{ }^{(l)}$, if the condition for the convergence of the iterative calculation process is not met 


$$
\left|\sum_{t=1}^{24} Q_{j t}\left(P_{j t}\right)-Q_{j 3}\right| \leq \varepsilon_{\Delta Q}, \quad j=1,2, \ldots, m
$$

is carried out by the following expression

$$
\lambda_{j}^{(1)}=\left\{\begin{array}{lll}
\lambda_{j}^{(0)}+\gamma_{j}^{(0)} \Delta \lambda_{j} & \text { if } & \Delta Q_{j}^{(0)}>0, \\
\lambda_{j}^{(0)}-\gamma_{j}^{(0)} \Delta \lambda_{j} & \text { if } & \Delta Q_{j}^{(0)}<0,
\end{array}\right.
$$

where $\Delta \lambda_{\mathrm{j}}$ is a small positive number - the increment of the factor; $\gamma_{j}^{(o)}=1$ is the scale factor, which is found in subsequent iterations as $\gamma_{j}^{(k)}=2 \gamma_{j}^{(k-1)}$.

In subsequent $t$ th iterations for those HPPs, in which the change in the sign of the residual $\Delta \mathrm{Qj}$ has not yet been observed in the previous iterations, is found by expression (9), where instead of $\lambda_{j}{ }^{(0)}, \gamma_{j}^{(0)}$ uses $\lambda_{j}^{(t-1)}$ and $\gamma_{j}^{(t-1)}$, appropriately, and HPP, in which a change in the sign of the residual $\Delta Q_{j}$ has already been observed, they are calculated by the chord method using the expression

$$
\lambda_{j}^{(t)}=\lambda_{j}^{\prime \prime}-\frac{\lambda_{j}^{\prime \prime}-\lambda_{j}^{\prime}}{\Delta Q_{j}^{\prime \prime}-\Delta Q_{j}^{\prime}} \cdot \Delta Q_{j}^{\prime} .
$$

In (10), individual parameters are determined according to the following conditions:

if the first change in the sign of the residual $\Delta Q_{j}$ occurred in the $t-1$ th iteration, then

$\Delta Q_{j}{ }^{\prime}=\Delta Q_{j}{ }^{(t-2)}, \Delta Q_{j}{ }^{\prime}=\Delta Q_{j}{ }^{(t-1)}, \quad \lambda_{j}{ }^{\prime}=\lambda_{j}{ }^{(t-2)}, \quad \lambda_{j}{ }^{\prime}=\lambda_{j}{ }^{(t-1)}$;

if the first change in the sign of the residual $\Delta Q_{j}$ occurred earlier - before the $t-1$ th iteration, where the residual and the factor had the values of $\Delta Q_{j}^{(f)}$ and $\lambda_{j}^{(f)}$, then in the case of $\Delta Q_{j}^{(t-1)} \cdot \Delta Q_{j}^{(f)}<0$ it is taken $\Delta Q_{j}{ }^{\prime}=$ $\Delta Q_{j}^{(f)}, \Delta Q_{j}{ }^{\prime \prime}=\Delta Q_{j}^{(t-1)}, \quad \lambda_{j}{ }^{\prime}=\lambda_{j}{ }^{(f)}, \quad \lambda_{j}{ }^{\prime \prime}=\lambda_{j}{ }^{(t-l)}$; in the case of $\Delta Q_{j}^{(t-1)} \cdot \Delta Q_{j}^{(f)}>0 \quad$ it is taken $\Delta Q_{j}{ }^{\prime \prime}=$ $\Delta Q_{j}{ }^{(t-l)}, \quad \lambda_{j}{ }^{\prime \prime}=\lambda_{j}{ }^{(t-l)}$ is taken, and the values of $\Delta Q_{j}{ }^{\prime}, \quad \lambda_{j}{ }^{\prime}$ remain unchanged - such as when performing previous iteration.

The minimization of function (7) subject to constraints (2) - (5) is carried out by the methods proposed in [17, $18]$.

\section{Results and discussion}

The efficiency of the described algorithm was researched, in particular, on the example of the optimal coverage of the load graph of the power system, given in Table 1, by two TPPs and three HPPs. The characteristics of relative increments (CRI) of the equivalent fuel consumption in TPPs and water consumption in HPPs are given in Tables 2 and 3, respectively. In these tables $B_{i}^{\text {min }}$ is the consumption of equivalent fuel in the $i$ th TPP at its minimum load; $Q_{j}^{\text {min }}$ is a water consumption in the $j$ th HPP at its minimum load; $Q_{\text {jav.day. }}$ is the given average daily water consumption in the $j$ th HPP. It is assumed that the given CRIs correspond to the optimal compositions of the operating units in the corresponding plants during the day.

Table 1. Daily load schedule of power system.

\begin{tabular}{|c|c|c|c|c|c|c|c|c|c|c|c|c|}
\hline$t, h$. & 1 & 2 & 3 & 4 & 5 & 6 & 7 & 8 & 9 & 10 & 11 & 12 \\
\hline$P_{L}, \mathrm{MW}$ & 2850 & 2750 & 2700 & 2750 & 2850 & 2900 & 3000 & 3100 & 3150 & 3200 & 3250 & 3200 \\
\hline$t, h$. & 13 & 14 & 15 & 16 & 17 & 18 & 19 & 20 & 21 & 22 & 23 & 24 \\
\hline$P_{L}, \mathrm{MW}$ & 3000 & 2950 & 3050 & 3100 & 3250 & 3400 & 3500 & 3600 & 3550 & 3400 & 3250 & 3000 \\
\hline
\end{tabular}

Table 2. CRI of equivalent fuel consumption at TPP.

\begin{tabular}{|c|c|c|c|c|c|c|c|c|c|c|}
\hline \multirow{2}{*}{$\begin{array}{c}\text { HPP-1, } \\
\mathrm{Q}_{1} \mathrm{~min}= \\
104 \mathrm{~m}^{3} / \mathrm{s} . \\
\mathrm{Q}_{1 \text { av.day. }}= \\
570 \mathrm{~m}^{3} / \mathrm{s} .\end{array}$} & $\mathrm{P}_{\Gamma 1,}, \mathrm{MW}$ & $\begin{array}{c}0 \\
601 \\
\end{array}$ & $\begin{array}{l}323 \\
604 \\
\end{array}$ & $\begin{array}{l}579 \\
607 \\
\end{array}$ & $\begin{array}{l}582 \\
611 \\
\end{array}$ & $\begin{array}{l}586 \\
614 \\
\end{array}$ & $\begin{array}{l}589 \\
620 \\
\end{array}$ & 592 & 595 & 598 \\
\hline & $q_{1} \frac{m^{3}}{M W \cdot h}$ & $\begin{array}{c}1.128 \\
1.2072\end{array}$ & $\begin{array}{c}1.1368 \\
1.216\end{array}$ & $\begin{array}{l}1.1456 \\
1.2248\end{array}$ & $\begin{array}{l}1.1544 \\
1.2336\end{array}$ & $\begin{array}{l}1.1632 \\
1.2424\end{array}$ & $\begin{array}{c}1.172 \\
1.26\end{array}$ & 1.1808 & 1.1896 & 1.1984 \\
\hline \multirow{2}{*}{$\begin{array}{c}\mathrm{HPP}-2, \\
\mathrm{Q}_{2}{ }^{\mathrm{min}}= \\
20 \mathrm{~m}^{3} / \mathrm{s} . \\
\mathrm{Q}_{\text {2av.day. }}= \\
200 \mathrm{~m}^{3} / \mathrm{s} .\end{array}$} & $\mathrm{P}_{\Gamma 2, \mathrm{MW}}$ & $\begin{array}{c}0 \\
1200 \\
\end{array}$ & 100 & 200 & 300 & 400 & 500 & 600 & 800 & 1000 \\
\hline & $q_{2}, \frac{m^{3}}{M W \cdot h}$ & $\begin{array}{c}0.2 \\
12.0\end{array}$ & 0.3 & 0.4 & 0.5 & 0.7 & 1.0 & 2.0 & 4.3 & 8.0 \\
\hline \multirow{2}{*}{$\begin{array}{c}\mathrm{HPP}-3, \\
\mathrm{Q}_{3} \mathrm{~min}= \\
30 \mathrm{~m}^{3} / \mathrm{s} . \\
\mathrm{Q}_{3 \text { av.day. }}= \\
160 \mathrm{~m}^{3} / \mathrm{s} .\end{array}$} & $\mathrm{P}_{\Gamma 3,}, \mathrm{MW}$ & 0 & 160 & 180 & 196 & 390 & 400 & 440 & 480 & \\
\hline & $q_{3}, \frac{m^{3}}{M W \cdot h}$ & 0.1 & 0.14 & 0.21 & 0.42 & 0.47 & 0.55 & 1.27 & 4.03 & \\
\hline
\end{tabular}

\begin{tabular}{|c|c|c|c|c|c|c|c|c|c|}
\hline $\begin{array}{c}\text { TЭС-1 } \\
B_{1}{ }^{\min }=250,5 \\
t . f . e . / h .\end{array}$ & $\mathrm{P}_{\mathrm{T} 1, \mathrm{MW}}$ & 750 & 1200 & 1350 & 1355 & 1500 & & & \\
\cline { 2 - 10 } & $b_{1,}, \frac{t . f . e .}{M W \cdot h}$ & 0.295 & 0.296 & 0.297 & 0.3 & 3.3001 & & & \\
\hline $\begin{array}{c}\text { TЭС-2 } \\
2_{2}^{\max }=114,8 \\
t . f . e . / h .\end{array}$ & $\mathrm{P}_{\mathrm{T} 2, \mathrm{MW}}$ & 400 & 565 & 583 & 595 & 647 & 715 & 759 & 790 \\
\cline { 2 - 9 } & $b_{2,}, \frac{t . f . e .}{M W \cdot h}$ & 0.2705 & 0.2836 & 0.2967 & 0.3098 & 0.3229 & 0.336 & 0.3491 & 0.3622 \\
\hline
\end{tabular}

Table 3. Characteristics of the relative sprouting of water discharge of HPP. 
To compare the optimization results according to the proposed algorithm, Table 4 shows the optimal result obtained by the classical method based on the equality of the relative increases in fuel costs, with the determination of the factors $\lambda_{\mathrm{j}}$ by a simple selection. We accept this result as a reference. In this case, the optimal result was obtained in 1385 iterations.

Table 4. Reference optimum result.

\begin{tabular}{|c|c|c|c|c|c|c|}
\hline \multirow[t]{2}{*}{$t, h$. } & \multirow[t]{2}{*}{$P_{L}, \mathrm{MW}$} & \multicolumn{5}{|c|}{ Optimal loads of power plant, MW } \\
\hline & & TPP-1 & TPP-2 & HPP-1 & HPP-2 & HPP-3 \\
\hline 1 & 2850 & 1140.39 & 581,86 & 294,72 & 417,97 & 415,06 \\
\hline 2 & 2750 & 1064.54 & 581,62 & 270,97 & 417,83 & 415,04 \\
\hline 3 & 2700 & 1026.62 & 581,51 & 259,09 & 417,76 & 415,03 \\
\hline 4 & 2750 & 1064.54 & 581,62 & 270,97 & 417,83 & 415,04 \\
\hline 5 & 2850 & 1140.39 & 581,86 & 294,72 & 417,97 & 415,06 \\
\hline 6 & 2900 & 1178.31 & 581,97 & 305,60 & 418,04 & 415,08 \\
\hline 7 & 3000 & 1239.43 & 582,40 & 344,74 & 418,31 & 415,13 \\
\hline 8 & 3100 & 1296.23 & 582,92 & 387,04 & 418,63 & 415,18 \\
\hline 9 & 3150 & 1324.66 & 583,12 & 408,21 & 418,79 & 415,21 \\
\hline 10 & 3200 & 1350.08 & 583.32 & 432.38 & 418.97 & 415.25 \\
\hline 11 & 3250 & 1350.80 & 583.72 & 480.83 & 419.34 & 415.31 \\
\hline 12 & 3200 & 1350.08 & 583.32 & 432.38 & 418.97 & 415.25 \\
\hline 13 & 3000 & 1239.43 & 582.40 & 344.74 & 418.31 & 415.13 \\
\hline 14 & 2950 & 1211.02 & 582.14 & 323.59 & 418.15 & 415.10 \\
\hline 15 & 3050 & 1267.83 & 582.66 & 365.89 & 418.47 & 415.16 \\
\hline 16 & 3100 & 1296.23 & 582.92 & 387.04 & 418.63 & 415.18 \\
\hline 17 & 3250 & 1350.80 & 583.72 & 480.83 & 419.34 & 415.31 \\
\hline 18 & 3400 & 1395.56 & 586.05 & 581.18 & 421.51 & 415.71 \\
\hline 19 & 3500 & 1495.33 & 586.11 & 581.27 & 421.56 & 415.72 \\
\hline 20 & 3600 & 1500.00 & 634.59 & 608.38 & 438.28 & 418.75 \\
\hline 21 & 3550 & 1500.00 & 603.22 & 597.68 & 431.57 & 417.53 \\
\hline 22 & 3400 & 1395.56 & 586.05 & 581.18 & 421.51 & 415.71 \\
\hline 23 & 3250 & 1350.80 & 583.72 & 480.83 & 419.34 & 415.31 \\
\hline 24 & 3000 & 1239.43 & 582.40 & 344.74 & 418.31 & 415.12 \\
\hline \multicolumn{7}{|c|}{ Lagrange multipliers and average daily water consumption in HPP } \\
\hline \multicolumn{2}{|c|}{ HPP } & \multicolumn{3}{|c|}{$\lambda_{j}$} & \multicolumn{2}{|c|}{$Q_{\text {c.av.day. }}, \mathrm{m}^{3} / \mathrm{s}$. } \\
\hline \multicolumn{2}{|c|}{ HPP-1 } & \multicolumn{3}{|c|}{0.2604} & \multicolumn{2}{|c|}{570.03} \\
\hline \multicolumn{2}{|c|}{ HPP-2 } & \multicolumn{3}{|c|}{0.3924} & \multicolumn{2}{|c|}{199.80} \\
\hline \multicolumn{2}{|c|}{ HPP-3 } & \multicolumn{3}{|c|}{0.3603} & \multicolumn{2}{|c|}{159.84} \\
\hline
\end{tabular}

When obtaining the reference result, the accuracy of the calculation for the discrepancies of the water flow rates in the HPP is taken as $0.1 \%$ of the given average daily water flow rate, i.e. $\varepsilon_{\Delta Q}=0.1 \%$.

The experimental calculations have shown that at large values of the step $\Delta \lambda_{j}$, in some cases, the result can be obtained in a smaller number of iterations, and in the general case, the process may not converge or looping. In particular, when calculating with $\varepsilon_{\Delta \mathrm{Q}}=0.1 \%$ and a step of $\Delta \lambda_{i}=0.00005$, the calculation process did not converge to a solution - it looped, and at $\Delta \lambda_{\mathrm{i}}=0.00002$ it was ensured with the accepted accuracy. Table 5 shows the $\lambda_{\mathrm{j}}$ factors, the discrepancy between the average daily water consumption at the HPP $\Delta Q_{j}$ and the total consumption of the equivalent fuel in the TPP per day B, obtained as a result of the optimal coverage of daily load graph of the power system by the methods described in $[17,18]$ with the determination of values of $\lambda_{j}$ multipliers proposed here algorithm, where the optimal results are obtained in 3-4 iterations.

Table 5. The results of optimal coverage of daily load schedule of power systems with the choice of $\lambda_{j}$ by the combined algorithm.

\begin{tabular}{|c|c|c|c|c|c|c|c|c|}
\hline \multirow{2}{*}{$\begin{array}{l}\varepsilon \Delta Q, \quad \% \\
\text { from } Q_{j 3}\end{array}$} & \multirow{2}{*}{$\begin{array}{l}\text { Number } \\
\text { of } \\
\text { iterations }\end{array}$} & \multicolumn{2}{|c|}{ HPP-1 } & \multicolumn{2}{|c|}{ HPP-2 } & \multicolumn{2}{|c|}{ ГЭС-3 } & \multirow{2}{*}{$\begin{array}{c}B \\
\text { t.f.e. }\end{array}$} \\
\hline & & $\lambda_{1}$ & $\Delta Q_{1}, m^{3} / s$ & $\lambda_{2}$ & $\Delta Q_{2}, \mathrm{~m}^{3} / \mathrm{s}$ & $\lambda_{3}$ & $\Delta Q_{3}, \mathrm{~m}^{3} / \mathrm{s}$ & \\
\hline 1 & 3 & 0.26048 & -2.55 & 0.39390 & -0.89 & 0.35775 & 0.12 & 13809.69 \\
\hline 0.5 & 3 & 0.26048 & -2.38 & 0.39390 & -0.89 & 0.35798 & 0.09 & 13808.86 \\
\hline 0.1 & 4 & 0.26045 & -0.46 & 0.39239 & -0.18 & 0.35793 & 0.09 & 13799.76 \\
\hline
\end{tabular}

To analyze the convergence of the iterative optimization process, Table 6 shows the results obtained in iterations when choosing the multipliers by the proposed algorithm. 
Table 6. Changes in $\lambda_{j}$ multipliers and discrepancies in water flow rates during iterations.

\begin{tabular}{|c|c|c|c|c|c|c|}
\hline \multirow{2}{*}{ Number of iteration } & \multicolumn{2}{|c|}{ HPP-1 } & \multicolumn{2}{c|}{ HPP-2 } & \multicolumn{2}{c|}{ HPP-3 } \\
\cline { 2 - 7 } & $\lambda_{1}$ & $\Delta Q_{1}, \mathrm{~m}^{3} / s$. & $\lambda_{2}$ & $\Delta Q_{2}, \mathrm{~m}^{3} / \mathrm{s}$. & $\lambda_{3}$ & $\Delta Q_{3}, \mathrm{~m}^{3} / \mathrm{s}$ \\
\hline 0 & 0.2602 & 18.4 & 0.3939 & -0.9295 & 0.3880 & -2.787 \\
\hline 1 & 0.2702 & -232.202 & 0.3839 & 8.883 & 0.3780 & -1.0877 \\
\hline 2 & 0.26093 & -29.922 & 0.39295 & -0.268 & 0.37160 & -1.267 \\
\hline 3 & 0.26048 & -2.579 & 0.39269 & -0.307 & 0.35794 & 0.0967 \\
\hline 4 & 0.26045 & -0.63 & 0.39239 & -0.1759 & 0.35794 & 0.0941 \\
\hline
\end{tabular}

The results obtained allow us to conclude that the proposed algorithm for choosing the values of the factors $\lambda_{j}$ provides fast and reliable convergence of the iterative computational process.

It should be noted that in the problem under consideration, it is also important to correctly choose the permissible discrepancy of the water flow rate in the HPP (the accuracy of the calculation based on the daily water consumption in the HPP) $\varepsilon_{\Delta Q}$.

To study this issue, the optimization of the daily mode of the hydrothermal power system (in the example under consideration) was carried out at various values of $\varepsilon \Delta \mathrm{Q}$. Based on the results obtained, it has been established that for large values of $\varepsilon_{\Delta Q}$, the result can be obtained with lower total daily consumption of equivalent fuel in TPPs and large excessive consumption of water in HPPs. Such modes cannot be considered optimal, since the deviation of water flow rates in hydroelectric power plants from its preset values by large values violates the system-wide optimality due to the violation of the optimal regimes of reservoirs determined in advance in long-term planning. In this regard, for the permissible maximum values of $\varepsilon_{\Delta \mathrm{Q}}$, we can take those values at which the deviation of the total daily consumption of equivalent fuel in TPP from their values in the reference result does not exceed $0.1 \%$.

\section{Conclusion}

1. An algorithm for the optimal planning of short-term modes of hydrothermal power systems which characterized by effective calculation of indefinite Lagrange multipliers, which are costly or fuel equivalent of water consumption in hydroelectric power plants is proposed,

2. Calculation of indefinite Lagrange multipliers for the functions of imbalances of water flow rates on the basis of the proposed combined method allows to obtain an optimal solution of the problem with sufficient accuracy rather reliably and in a small number of iterations.

3. The proposed algorithm can be used by dispatching services for optimal planning of short-term modes of hydrothermal power systems.

\section{References}

1. H.F. Fazylov, T.X, Nasyrov, The steady-state modes of electric power systems and its optimization (Tashkent: Moliya, 1999). (in Russian).
2. de Queiroz, A. R. (2016). Renewable and Sustainable Energy Reviews, $\quad 62$ (2016). https://doi.org/10.1016/j.rser.2016.04.065

3. Zambon, R. C., Barros, M. T., Lopes, J. E. G., Barbosa, P. S., Francato, A. L., \& Yeh, W. W. G. Journal of Water Resources Planning and Management, $\quad \mathbf{1 3 8 ( 2 )}$ https://doi.org/10.1061/(ASCE)WR.19435452.0000149 .

4. Zambelli, M., Soares Filho, S., Toscano, A. E., Santos, E. D., \& Silva Filho, D. D. Controle \& Automac. Sociedade Brasileira de Automatica, 22(6) (2011). https://doi.org/10.1590/S010317592011000600005.

5. Li, C., Zhou, J., Lu, P., \& Wang, C. Short-term economic environmental hydrothermal scheduling using improved multi-objective gravitational search algorithm. Energy Conversion and Management, 89 (2015).

https://doi.org/10.1016/j.enconman.2014.09.063.

6. Zhou, J. Z., Liao, X., Ouyang, S., Zhang, R., \& Zhang, Y. C. International Journal of Electrical Power \& Energy Systems, 55 (2014). https://doi.org/10.1016/j.ijepes.2013.10.013

7. Mandal, K. K., \& Chakraborty, N. Expert Systems With Applications, 39(3) (2011). https://doi.org/10.1016/j.eswa.2011.09.032.

8. Wood Allen, J., Wollenberg Bruce, F., Sheble Gerald, B. Power Generation Operation and Control (John Wiley \& Sons, 1996).

9. Rodrigues, R.N., et al. Math. Probl. Eng. (2012).

10. Automation of dispatching management in electric power industry / Under general reduction of J.N.Rudenko and V.A.Semyonov (M: MEI, 2000). (In Russian).

11. Zhang, Huifeng, et al.Energy 122 (2017).

12. Fang, N., et al. Int. J. Electr. Power Energy Syst. 62 (2014).

13 Nallasivan, C., et al. In: 2006 IEEE Power India Conference. (2006).

14. Kumar, V.S., Mohan, M. Int. J. Electr. Power Energy Syst. 33 (4) (2011).

15. Samudi, C., et al. In: IEEE/PES Transmission and Distribution Conference and Exposition. IEEE. (2008).

16. Letun B.M., Shubin N.G. 3- International scientificpractical conference (October 13-16, 2008. vol. 1). (In Russian).

17. T. Gayibov, Proceedings of the 2011 3rd International Youth Conference on Energetics (IYCE) (Leiria, Portugal, 2011). 
18. Tulkin Gayibov, Sherxon Latipov, Bakhadir Uzakov. Rudenko International Conference "Methodological problems in reliability study of large energy systems" E3S Web of Conference, 139, 01086 (2019).

https://doi.org/10.1051/e3sconf/201913901086. 